\title{
FM12p: Focus Meeting 12 Poster Session
}

\section{Poster Presentations:}

FM12p.01 Electron impact excitation of Astrophysically Important C III Ion. Author(s): Kanti M Aggarwal, Francis P Keenan

FM12p.02 Dielectronic Satellite Lines of Fe XVII. Author(s): Peter Beiersdorfer, Gregory V Brown, Alexander Laska, Jaan K. Lepson

FM12p.03 Calculations of effective recombination coefficients for nebular astrophysics. Author(s): Xuan Fang, Xiaowei Liu, Pete J. Storey

FM12p.04 Atomic lines in multiwavelength spectral analysis of late-type stars. Author(s): Hugh Jones

FM12p.05 Charge Exchange Produced Emission of Carbon in the Iron M-shell Dominated 150-200 Extreme Ultraviolet Region. Author(s): Jaan K. Lepson, Peter Beiersdorfer, Manfred Bitter, A. Lane Roquemore, Robert Kaita

FM12p.06 The role of hydrogen collisions in non-LTE abundance analyses of aluminium. Author(s): Thomas Nordlander, Karin Lind

FM12p.07 Progress in Identifying Fe I Level Energies and Lines from Stellar Spectra. Author(s): Ruth Peterson

FM12p.08 Atomic Oscillator Strengths for Stellar Atmosphere Modeling. Author(s): Matthew Ruffoni, Juliet C Pickering

FM12p.09 Improved and Expanded Near-IR Oscillator Strengths for Fe-group Elements. Author(s): Michael Wood, Gillian Nave, Christopher Alan Sneden

FM12p.10 Improved wavelengths for $\mathrm{Fe} \mathrm{V}$ and $\mathrm{Ni} \mathrm{V}$ for analysis of spectra of white dwarf stellar stars. Author(s): Jacob Ward, Gillian Nave

FM12p.11 Wavelengths, energy levels and hyperfine structure of Mn II and Sc II. Author(s): Gillian Nave, Juliet C Pickering, Keeley I. M. Townley-Smith, . Hala

FM12p.12 New laboratory atomic data for neutral, singly and doubly ionised iron group elements for astrophysics applications. Author(s): Juliet C Pickering, Gillian Nave, Florence Liggins, Christian Clear, Matthew Ruffoni, Craig Sansonetti

FM12p.13 Report on the recent advances performed in the determination of radiative parameters for spectral lines of astrophysical interest in heavy elements. Author(s): Pascal Quinet

FM12p.14 A systematic and detailed investigation of radiative rates for forbidden transitions of astrophysical interest in doubly ionized iron peak elements. Author(s): Pascal Quinet, Vanessa Fivet, Manuel Bautista

FM12p.15 The Interstellar Abundance of Lead: Experimental Oscillator Strengths for $\mathrm{Pb}$ II $\lambda 1203$ and $\lambda 1433$ and New Detections of $\mathrm{Pb}$ II in the Interstellar Medium. Author(s): Adam Michael Ritchey, Negar Heidarian, Richard E. Irving, Steven R. Federman, David G. Ellis, Song Cheng, Larry J. Curtis, W. A. Furman

FM12p.16 High Energy Laboratory Astrophysics Experiments using electron beam ion traps and advanced light sources. Author(s): Gregory V Brown, Peter Beiersdorfer, Sven Bernitt, Sita Eberle, Natalie Hell, Caroline Kilbourne, Rich Kelley, Maurice Leutenegger, F. Scott Porter, Jan Rudolph, Rene Steinbrugge, Elmar Traebert, Jose R. Crespo-LopezUrritia 
FM12p.17 High-resolution oscillator strength measurements of the $\mathrm{v}^{\prime}=0,1$ bands of the B- X, C -X, and E- X systems in five isotopologues of carbon monoxide. Author(s): Glenn Stark, Alan Heays, James Lyons, Michelle Eidelsberg, Steve Federman, Jean Louis Lemaire, Nelson de Oliveira, Laurent Nahon

FM12p.18 Identifying New Molecules from Comparison of Herschel-HIFI Spectra with ab initio Computational Spectra. Author(s): Naseem Rangwala, Sean Colgan, Timothy J Lee, Xinchuan Huang, Ryan Fortenberry

FM12p.19 IR Line Lists of $\mathrm{SO}_{2}$ and $\mathrm{CO}_{2}$ Isotopologues for Atmospheric Modeling on Venus and Exoplanets. Author(s): Xinchuan Huang, David W Schwenke, Timothy J Lee

FM12p.21 Laser Induced Fluorescence Spectroscopy of Neutral and Ionized Polycyclic Aromatic Hydrocarbons in a Cosmic Simulation Chamber. Author(s): Salma Bejaoui, Farid Salama

FM12p.22 Infrared spectra of interstellar deuteronated PAHs. Author(s): Mridusmita Buragohain, Amit Pathak, Peter Sarre

FM12p.23 Synthesis of Pure and N-substituted Cyclic Hydrocarbons (e.g. Pyrimidine) via Gas-Phase Ion-Molecule Reactions. Author(s): Partha P Bera, Roberto Peverati, Martin Head-Gordon, Timothy J Lee

FM12p.24 Coronene and Pyrene (5,7)-member Ring Defects. Author(s): Silvia Oettl, Stefan Kimeswenger, Stefan E. Huber

FM12p.26 Structural Evolution of Interstellar Polycyclic Aromatic Hydrocarbons. Author(s): Mark Hammonds, Alessandra Candian, Tamami Mori, Fumihiko Usui, Takashi Onaka

FM12p.27 Laboratory Astrophysics Studies with the COSmIC Facility: Interstellar and Planetary Applications. Author(s): Farid Salama, Cesar S. Contreras, Ella SciammaO'Brien, Salma Bejaoui

FM12p.28 Interstellar Methanol from the Lab to Protoplanetary Disks. Author(s): Maria Drozdovskaya, Catherine Walsh, Ruud Visser, Daniel Harsono, Ewine van Dishoeck

FM12p.29 Organic molecules in ices and their release into the gas phase. Author(s): Edith Fayolle, Karin I Oberg, Robin Garrod, Ewine van Dishoeck, Mahesh Rajappan, Mathieu Bertin, Claire Romanzin, Xavier Michaut, Jean-Hugues Fillion

FM12p.30 Combining Laboratory and Observational Data to Elucidate the Pathway from Simple to Complex Chemistry. Author(s): Helen Jane Fraser, Aleksi Suutarinnen, Anita Dawes, Jennifer Noble

FM12p.31 Are interstellar ices porous, and how do the pores collapse? Author(s): Catherine Rachel Hill, Christian Mitterdorfer, Tristan G. A. Youngs, Natalia Pascual, Olivier Auriacombe, Thomas Loerting, Helen J. Fraser

FM12p.32 THz Time-Domain Spectroscopy of Interstellar Ice Analogs. Author(s): Sergio Ioppolo, Brett A McGuire, Xander de Vries, Brandon Carroll, Marco Allodi, Geoffrey Blake

FM12p.33 Evidence of amino acid precursors: C-N bond coupling in simulated interstellar $\mathrm{CO}_{2} / \mathrm{NH}_{3}$ ices. Author(s): Sasan Esmaili

FM12p.34 Untangling molecular signals of astrochemical ices in the THz: distinguishing amorphous, crystalline, and intramolecular modes with broadband $\mathrm{THz}$ spectroscopy. Author(s): Brett A McGuire, Sergio Ioppolo, Marco Allodi, Brandon Carroll, Geoffrey Blake

FM12p.36 The MAON model of Astronomical Unidentified Infrared Emission Bands. Author(s): Sun Kwok, Yong Zhang

FM12p.37 Molecules, Dust and Ices in Brown Dwarf Atmospheres. Author(s): Sandy Leggett, Mark Marley, Caroline Morley, Didier Saumon 
FM12p.38 The survival of PAHs and hydrocarbon nanoparticles in H II regions: theory and observations. Author(s): Elisabetta Micelotta, Marco Bocchio, Aurelie Remy-Ruyer, Melanie Kohler, Nathalie Ysard, Anthony Peter Jones

FM12p.39 Laboratory polarization and permittivity measurements to interpret dust polarimetric observations and in-situ radar studies. Significance for Rosetta mission at $67 \mathrm{P} /$ Churyumov-Gerasimenko. Author(s): Anny-Chantal Levasseur-Regourd, Yann Brouet, Edith Hadamcik, Essam Heggy, Dean Hines, Jeremie Lasue, Jean-Baptiste Renard

FM12p.40 Line survey observations of irradiated protostars - photo-destruction and evaporation. Author(s): Johan E Lindberg, Steven B Charnley, Jes K Jorgensen, Yoshimasa Watanabe, Suzanne E Bisschop, Nami Sakai, Satoshi Yamamoto

FM12p.41 Amorphous Silica- and Carbon- rich nano-templated surfaces as model interstellar dust surfaces for laboratory astrochemistry. Author(s): Natalia Pascual, Anita Dawes, Fernando Gonzlez-Posada, Neil Thompson, Dinko Chakarov, Nigel J Mason, Helen Jane Fraser

FM12p.42 Better Alternatives to Astronomical Silicate: Laboratory-Based Optical Functions of Chondritic/Solar Abundance Glass With Application to HD 161796. Author(s): Angela K Speck, Karly Pitman, Anne Hofmeister

FM12p.45 Infrared spectroscopy in the C-H stretching region towards embedded highmass young stellar objects in the Large Magellanic Cloud. Author(s): Takashi Shimonishi, Emmanuel Dartois, Takashi Onaka, Franois Boulanger

FM12p.46 Laboratory Analysis of Comet Samples. Author(s): Don Brownlee

FM12p.47 Fireball data analysis: bridging the gap between small solar system bodies and meteorite studies. Author(s): Maria Gritsevich, Manuel Moreno-Ibez, Daria Kuznetsova, Alexis Bouquet, Josep Trigo-Rodrguez, Jouni Peltoniemi, Detlef Koschny

FM12p.48 Origin of the Earths's Moon and Neptune's Triton. Author(s): Fred Johnson

FM12p.49 The Evolution of the Sun and the Planetary System. Author(s): Fred Johnson

FM12p.50 The Origins of the Planetary Satellites. Author(s): Fred Johnson

FM12p.52 Cosmological Plasma Instabilities - Plasma Physics at the Boundary. Author(s): Avery E Broderick

FM12p.54 Laboratory experiments investigating magnetic field production via the Weibel instability in interpenetrating plasma flows. Author(s): Channing Huntington, Frederico Fiuza, James Steven Ross, Alex Zylstra, Brad Pollock, R. Paul Drake, Dustin Froula, Gianluca Gregori, Nathan Kugland, Carolyn Kuranz, Matthew Levy, Chikang Li, Jena Meinecke, Richard Petrasso, Bruce Remington, Dmitri Ryutov, Youichi Sakawa, Anatoly Spitkovsky, Hideke Takabe, David Turnbull, Hye-Sook Park

FM12p.55 High performance computing (HPC) simulations of laboratory experiments probing astrophysical processes. Author(s): Giovanni Lapenta

FM12p.56 Studying counterstreaming high velocity plasma flows relevant to astrophysical collisionless shock. Author(s): James Steven Ross, Peter Amendt, Laurent Divol, Brad Pollock, Bruce Remington, Dmitri Ryutov, Wojciech Rozmus, David Turnbull, Dustin Froula, taichi morita, Youichi Sakawa, Hideke Takabe, R. Paul Drake, Carolyn C Kuranz, Gianluca Gregori, Jena Meinecke, Michel Koenig, Anatoly Spitkovsky, Hye-Sook Park

FM12p.57 Laboratory formation of a scaled protostellar jet by coaligned poloidal magnetic field: recent results and new exeprimental studies. Author(s): Tommaso Vinci, Guilhem Revet, Drew Higginson, Jrome Bard, K. Burdonov, Sophia Chen, D. Khagani, B. Khiar, K. Naughton, S. Pikuz, Caterina Riconda, R. Riquier, A. Soloviev, O. Willi, O. Portugall, Henry Pepin, Andrea Ciardi, Julien Fuchs, Bruno Albertazzi 
FM12p.58 Laboratory experiments on Radiative Shocks relevant to Stellar Accretion. Author(s): Uddhab Chaulagain

FM12p.60 Modeling the interaction of solar wind with a dipole magnetic field with intense lasers. Author(s): Jiayong Zhong

FM12p.61 Merged Beams Studies for Astrobiology. Author(s): Daniel Wolf Savin, Aodh P. O'Connor, Nathalie de Ruette, Kenneth Miller, Julia Stuetzel, Xavier Urbain

FM12p.62 Science Goals and Laboratory Astrophysics Needs of the James Webb Space Telescope. Author(s): George Sonneborn

FM12p.63 Entoto Twining Telescopes: first robotic facilities in East Africa for astronomy research. Author(s): Solomon Belay Tessema 